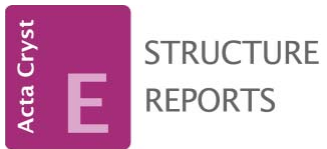

OPEN $\curvearrowright$ ACCESS

ISSN 1600-5368

\section{Crystal structure of dichlorido $\left(2,2^{\prime}: 6^{\prime}, 2^{\prime \prime}\right.$ - terpyridine- $\left.\kappa^{3} N, N^{\prime}, N^{\prime \prime}\right)$ zinc: a redeter- mination}

\author{
Cheng-Cheng Kong, Jia-Zheng Zhou, Jian-Hua Yu and \\ Sheng-Li Li*
}

Deparment of Chemistry, Anhui University, Hefei 230039, Peoples Republic of China, Key Laboratory of Functional Inorganic Materials, Chemistry, Hefei 230039, People's Republic of China. *Correspondence e-mail: Isl1968@ahu.edu.cn

Received 21 October 2014; accepted 27 October 2014

Edited by M. Weil, Vienna University of Technology, Austria

The crystal structure of the title compound, $\left[\mathrm{ZnCl}_{2}\left(\mathrm{C}_{15} \mathrm{H}_{11} \mathrm{~N}_{3}\right)\right]$, was redetermined based on modern CCD data. In comparison with the previous determination from photographic film data [Corbridge \& Cox (1956). J. Chem. Soc. 159, 594-603; Einstein \& Penfold (1966). Acta Cryst. 20, 924-926], all non-H atoms were refined with anisotropic displacement parameters, leading to a much higher precision in terms of bond lengths and angles [e.g. $\mathrm{Zn}-\mathrm{Cl}=2.2684$ (8) and 2.2883 (11) compared to 2.25 (1) and 2.27 (1) $\AA$ ]. In the title molecule, the $\mathrm{Zn}^{\mathrm{II}}$ atom is fivecoordinated in a distorted square-pyramidal mode by two $\mathrm{Cl}$ atoms and by the three $\mathrm{N}$ atoms from the $2,2^{\prime}: 6^{\prime}, 2^{\prime \prime}$-terpyridine ligand. The latter is not planar and shows dihedral angles between the least-squares planes of the central pyridine ring and the terminal rings of $3.18(8)$ and $6.36(9)^{\circ}$. The molecules in the crystal structure pack with $\pi-\pi$ interactions [centroidcentroid distance $=3.655(2) \AA$ ] between pyridine rings of neighbouring terpyridine moieties. These, together with intermolecular $\mathrm{C}-\mathrm{H} \cdots \mathrm{Cl}$ interactions, stablize the threedimensional structure.

Keywords: crystal structure; redetermination; $2,2^{\prime}: 6^{\prime}, 2^{\prime \prime}$-terpyridine; zinc complex; $\pi-\pi$ interactions.

CCDC reference: 1029855

\section{Related literature}

The title compound is dimorphic, with one polymorph (form I) crystallizing in space group No. 15, and the second polymorph (type II) crystallizing in space group No. 14 (Corbridge \& Cox, 1956). The crystal structure of the title compound was originally determined by Corbridge \& Cox (1956) from photographic data (final $R$ value $=0.24$ ) and was later re- refined by Einstein \& Penfold (1966) based on the original intensity data but using more advanced least-squares procedures $(R=0.14)$. In both reports, the setting in $P 2_{1} / a$ of space group No. 14 was used. For background to terpyridine-based materials, see: Fermi et al. (2014); Song et al. (2014). For the biocompatibility of zinc compounds, see: Gao et al. (2009).

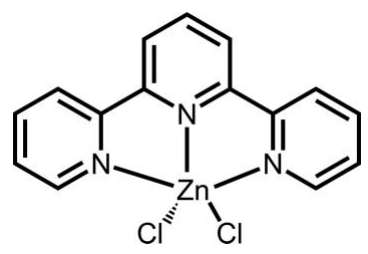

\section{Experimental}

2.1. Crystal data

$\left[\mathrm{ZnCl}_{2}\left(\mathrm{C}_{15} \mathrm{H}_{11} \mathrm{~N}_{3}\right)\right]$

$M_{r}=369.54$

Monoclinic, $P 2_{1} / c$

$a=10.950(5) \AA$

$b=8.250(5) \AA$

$c=16.216(5) \AA$

$\beta=93.911(5)^{\circ}$

$$
\begin{aligned}
& V=1461.5(12) \AA^{3} \\
& Z=4 \\
& \text { Mo } K \alpha \text { radiation } \\
& \mu=2.04 \mathrm{~mm}^{-1} \\
& T=298 \mathrm{~K} \\
& 0.30 \times 0.20 \times 0.20 \mathrm{~mm}
\end{aligned}
$$

\subsection{Data collection \\ Bruker SMART CCD diffractometer \\ Absorption correction: multi-scan (SADABS; Bruker, 2002) \\ 9990 measured reflections 2564 independent reflections 2404 reflections with $I>2 \sigma(I)$ $R_{\text {int }}=0.017$}

$T_{\min }=0.580, T_{\max }=0.686$

2.3. Refinement

$R\left[F^{2}>2 \sigma\left(F^{2}\right)\right]=0.020$

$w R\left(F^{2}\right)=0.056$

$S=1.05$

2564 reflections

190 parameters

$\mathrm{H}$-atom parameters constrained

$\Delta \rho_{\max }=0.24{\mathrm{e} \AA^{-3}}^{-3}$

$\Delta \rho_{\min }=-0.24$ e $\AA^{-3}$

Table 1

Hydrogen-bond geometry $\left(\AA,^{\circ}\right)$.

\begin{tabular}{lllll}
\hline$D-\mathrm{H} \cdots A$ & $D-\mathrm{H}$ & $\mathrm{H} \cdots A$ & $D \cdots A$ & $D-\mathrm{H} \cdots A$ \\
\hline $\mathrm{C} 4-\mathrm{H} 4 \cdots \mathrm{Cl}^{\mathrm{i}}$ & 0.93 & 2.68 & $3.518(2)$ & 151 \\
$\mathrm{C} 13-\mathrm{H} 13 \cdots \mathrm{Cl} 2^{\mathrm{ii}}$ & 0.93 & 2.81 & $3.686(2)$ & 158 \\
\hline
\end{tabular}

Symmetry codes: (i) $-x+1,-y+1,-z$; (ii) $-x,-y,-z$.

Data collection: SMART (Bruker, 2002); cell refinement: SAINT (Bruker, 2002); data reduction: $S A I N T$; program(s) used to solve structure: SHELXS97 (Sheldrick, 2008); program(s) used to refine structure: SHELXL97 (Sheldrick, 2008); molecular graphics: SHELXTL (Sheldrick, 2008) and DIAMOND (Brandenburg, 2006); software used to prepare material for publication: SHELXTL (Sheldrick, 2008).

\section{Acknowledgements}

This work was supported by the National Natural Science Foundation of China (51372003), Anhui Provincial Natural 
Science Foundation (1308085MB24) and the Educational Commission of Anhui Province of China (KJ2012A025).

Supporting information for this paper is available from the IUCr electronic archives (Reference: WM5082).

\section{References}

Brandenburg, K. (2006). DIAMOND. Crystal Impact GbR, Bonn, Germany.
Bruker (2002). SMART, SAINT and SADABS. Bruker AXS Inc., Madison, Wisconsin, USA.

Corbridge, D. E. C. \& Cox, E. G. (1956). J. Chem. Soc. pp. 594-603.

Einstein, F. W. B. \& Penfold, B. R. (1966). Acta Cryst. 20, 924-926.

Fermi, A., Bergamini, G., Roy, M., Gingras, M. \& Ceroni, P. (2014). J. Am. Chem. Soc. 136, 6395-6400.

Gao, Y. H., Wu, J. Y., Li, Y. M., Sun, P. P., Zhou, H. P., Yang, J. X., Zhang, S. Y., Jin, B. K. \& Tian, Y. P. (2009). J. Am. Chem. Soc. 131, 5208-5213.

Sheldrick, G. M. (2008). Acta Cryst. A64, 112-122.

Song, S. G., Xue, Y. H., Feng, L. F., Elbatal, H., Wang, P. S., Moorefield, C. N., Newkome, G. R. \& Dai, L. (2014). Angew. Chem. Int. Ed. 53, 1415-1419. 


\section{supporting information}

Acta Cryst. (2014). E70, m382-m383 [doi:10.1107/S1600536814023605]

\section{Crystal structure of dichlorido(2,2':6', $2^{\prime \prime}$-terpyridine- $\left.\kappa^{3} N, N^{\prime}, N^{\prime \prime}\right)$ zinc: a redetermination}

\section{Cheng-Cheng Kong, Jia-Zheng Zhou, Jian-Hua Yu and Sheng-Li Li}

\section{S1. Experimental}

A solution of 2,2':6', $2^{\prime \prime}$-terpyridine $(0.23 \mathrm{~g}, 1 \mathrm{mmol})$ in acetonitrile $(20 \mathrm{ml})$ was mixed with a solution of zinc chloride $(0.14 \mathrm{~g}, 1 \mathrm{mmol})$ in methanol $(5 \mathrm{ml})$ and refluxed for $4 \mathrm{~h}$. The reaction mixture was the cooled to room temperature and filtered into a large test tube. Colorless crystals were obtained at room temperature after two weeks. Yield: $75 \%$.

\section{S2. Refinement}

All hydrogen atoms were placed in geometrically idealized positions and constrained to ride on their parent atoms, with C $-\mathrm{H}=0.93 \AA$ and $U_{\text {iso }}(\mathrm{H})=1.2 U_{\text {eq }}(\mathrm{C})$.

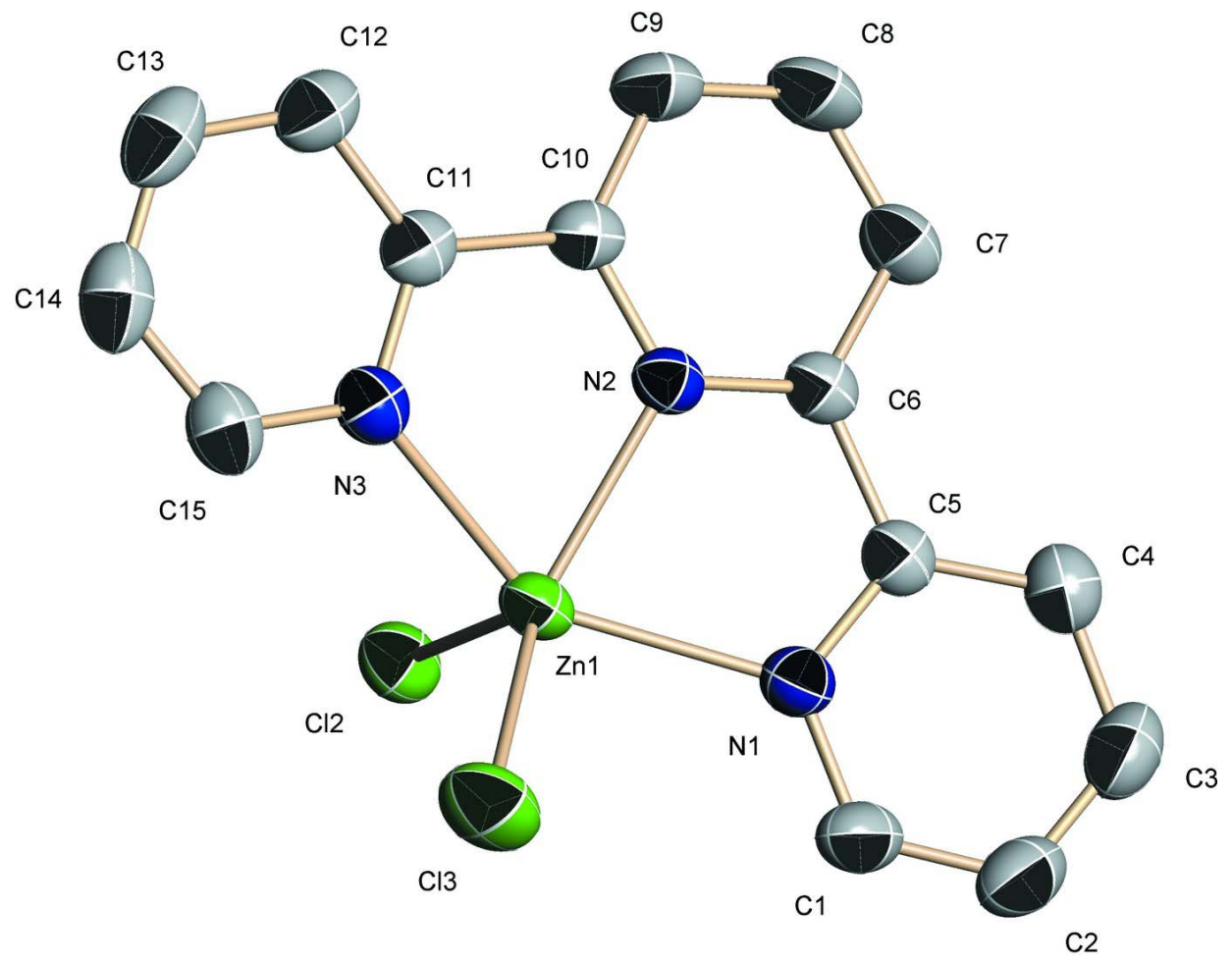

\section{Figure 1}

The molecular structure of (I), with the atom-numbering scheme. Displacement ellipsoids are drawn at the 50\% probability level. 


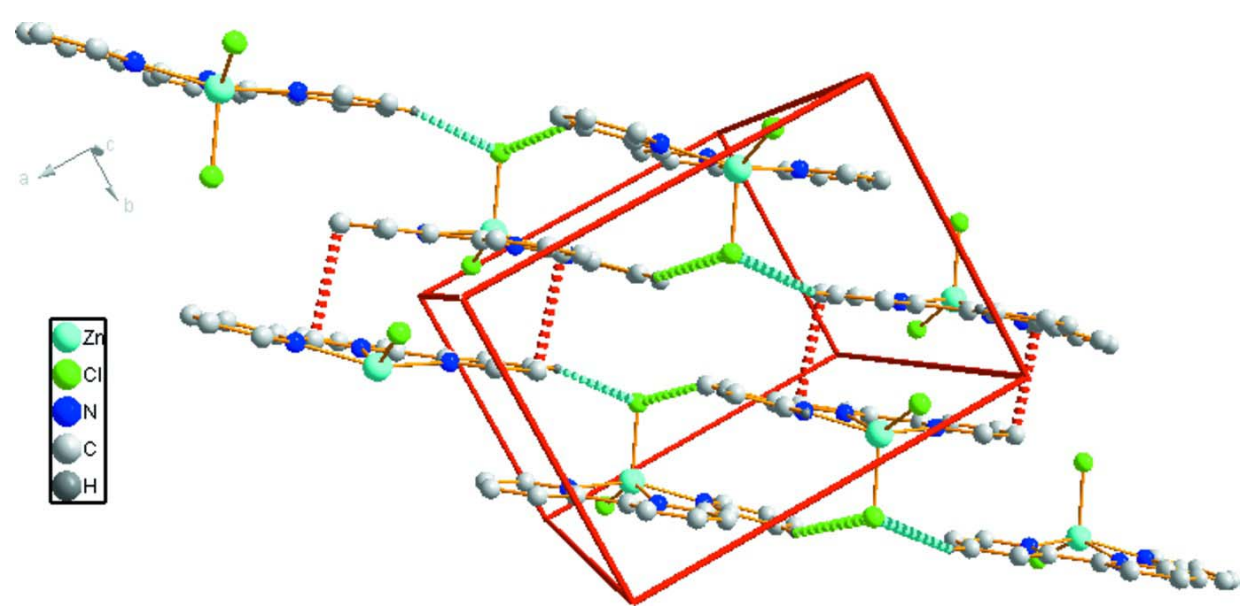

\section{Figure 2}

The arrangement of the molecules in the crystal structure of (I), showing $\pi-\pi$ interactions (dashed red lines) and C$\mathrm{H} \cdots \mathrm{Cl}$ hydrogen bonds (dashed green and turquoise lines).

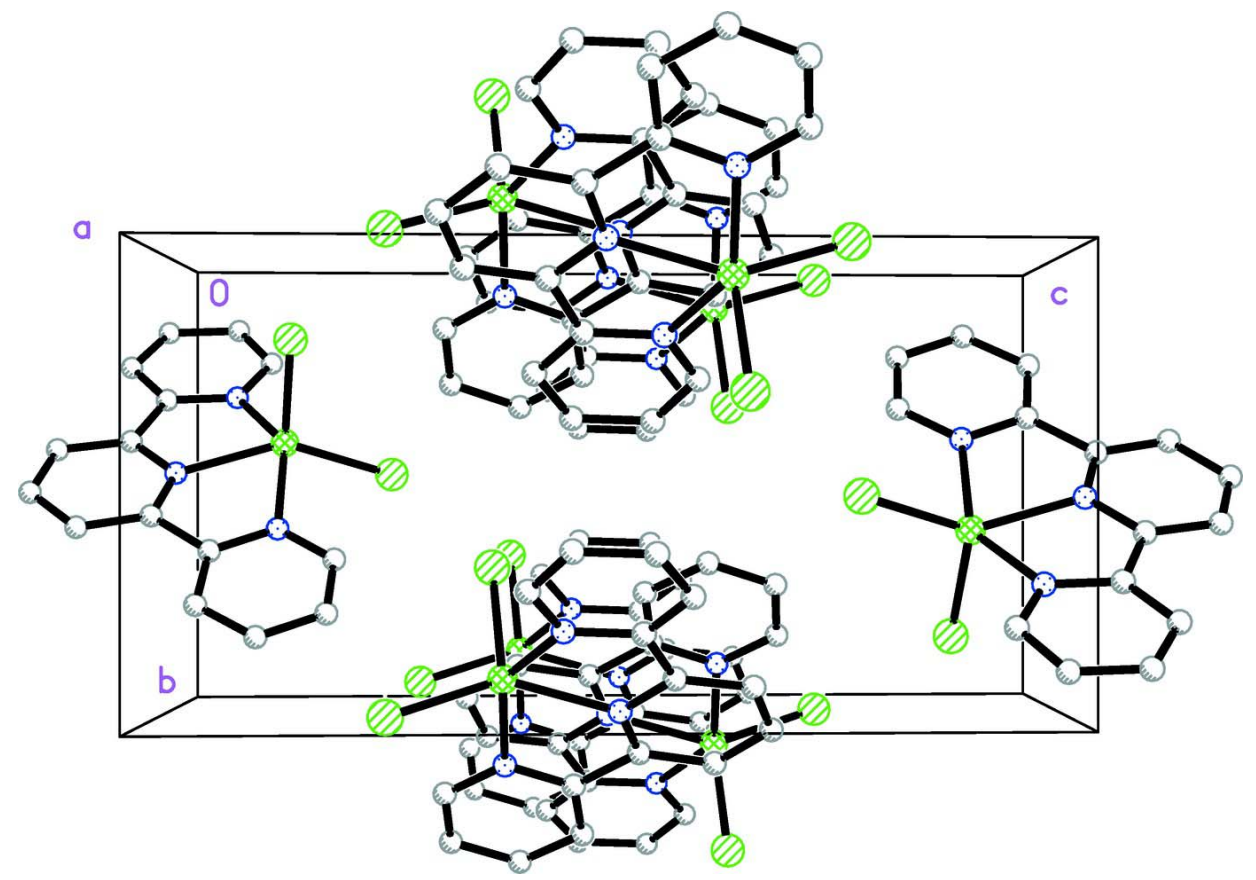

Figure 3

Packing diagram of (I). All $\mathrm{H}$ atoms have been omitted for clarity.

Dichlorido $\left(2,2^{\prime}: 6^{\prime}, 2^{\prime \prime}\right.$-terpyridine- $\left.\kappa^{3} N, N^{\prime}, N^{\prime \prime}\right)$ zinc

\section{Crystal data}

$\left[\mathrm{ZnCl}_{2}\left(\mathrm{C}_{15} \mathrm{H}_{11} \mathrm{~N}_{3}\right)\right]$

$M_{r}=369.54$

Monoclinic, $P 2{ }_{1} / c$

Hall symbol: -P $2 \mathrm{ybc}$

$a=10.950(5) \AA$

$b=8.250(5) \AA$

$c=16.216(5) \AA$ $\beta=93.911(5)^{\circ}$

$V=1461.5(12) \AA^{3}$

$Z=4$

$F(000)=744$

$D_{\mathrm{x}}=1.679 \mathrm{Mg} \mathrm{m}^{-3}$

Mo $K \alpha$ radiation, $\lambda=0.71069 \AA$

Cell parameters from 6593 reflections 
$\theta=2.8-26.9^{\circ}$

$\mu=2.04 \mathrm{~mm}^{-1}$

$T=298 \mathrm{~K}$

\section{Data collection}

\section{Bruker SMART CCD}

diffractometer

Radiation source: fine-focus sealed tube

Graphite monochromator

$\varphi$ and $\omega$ scans

Absorption correction: multi-scan

(SADABS; Bruker, 2002)

$T_{\min }=0.580, T_{\max }=0.686$

\section{Refinement}

Refinement on $F^{2}$

Least-squares matrix: full

$R\left[F^{2}>2 \sigma\left(F^{2}\right)\right]=0.020$

$w R\left(F^{2}\right)=0.056$

$S=1.05$

2564 reflections

190 parameters

0 restraints

Primary atom site location: structure-invariant direct methods
Block, colorless

$0.30 \times 0.20 \times 0.20 \mathrm{~mm}$

9990 measured reflections

2564 independent reflections

2404 reflections with $I>2 \sigma(I)$

$R_{\text {int }}=0.017$

$\theta_{\max }=25.0^{\circ}, \theta_{\min }=1.9^{\circ}$

$h=-13 \rightarrow 12$

$k=-9 \rightarrow 9$

$l=-18 \rightarrow 19$

Secondary atom site location: difference Fourier map

Hydrogen site location: inferred from neighbouring sites

$\mathrm{H}$-atom parameters constrained

$w=1 /\left[\sigma^{2}\left(F_{\mathrm{o}}^{2}\right)+(0.0322 P)^{2}+0.4083 P\right]$

where $P=\left(F_{\mathrm{o}}{ }^{2}+2 F_{\mathrm{c}}{ }^{2}\right) / 3$

$(\Delta / \sigma)_{\max }<0.001$

$\Delta \rho_{\max }=0.24$ e $\AA^{-3}$

$\Delta \rho_{\min }=-0.24$ e $\AA^{-3}$

\section{Special details}

Geometry. All e.s.d.'s (except the e.s.d. in the dihedral angle between two 1.s. planes) are estimated using the full covariance matrix. The cell e.s.d.'s are taken into account individually in the estimation of e.s.d.'s in distances, angles and torsion angles; correlations between e.s.d.'s in cell parameters are only used when they are defined by crystal symmetry. An approximate (isotropic) treatment of cell e.s.d.'s is used for estimating e.s.d.'s involving 1.s. planes.

Refinement. Refinement of $F^{2}$ against ALL reflections. The weighted $R$-factor $w R$ and goodness of fit $S$ are based on $F^{2}$, conventional $R$-factors $R$ are based on $F$, with $F$ set to zero for negative $F^{2}$. The threshold expression of $F^{2}>\sigma\left(F^{2}\right)$ is used only for calculating $R$-factors $(\mathrm{gt})$ etc. and is not relevant to the choice of reflections for refinement. $R$-factors based on $F^{2}$ are statistically about twice as large as those based on $F$, and $R$-factors based on ALL data will be even larger.

Fractional atomic coordinates and isotropic or equivalent isotropic displacement parameters $\left(\AA^{2}\right)$

\begin{tabular}{lllll}
\hline & $x$ & $y$ & $z$ & $U_{\text {iso }} * / U_{\text {eq }}$ \\
\hline Zn1 & $0.217983(18)$ & $0.40487(2)$ & $0.117728(11)$ & $0.03587(8)$ \\
C12 & $0.33535(4)$ & $0.17571(5)$ & $0.13148(3)$ & $0.04477(12)$ \\
C13 & $0.13940(5)$ & $0.47642(7)$ & $0.23837(3)$ & $0.06000(15)$ \\
N1 & $0.35875(14)$ & $0.59791(17)$ & $0.11822(9)$ & $0.0384(3)$ \\
N2 & $0.22895(12)$ & $0.47354(16)$ & $-0.00704(8)$ & $0.0324(3)$ \\
N3 & $0.05709(13)$ & $0.29671(17)$ & $0.05158(9)$ & $0.0385(3)$ \\
C4 & $0.48010(17)$ & $0.7621(2)$ & $0.03495(12)$ & $0.0471(4)$ \\
H4 & 0.5007 & 0.7944 & -0.0173 & $0.057 *$ \\
C5 & $0.39054(15)$ & $0.6462(2)$ & $0.04352(10)$ & $0.0353(4)$ \\
C10 & $0.15500(16)$ & $0.40050(19)$ & $-0.06497(11)$ & $0.0352(4)$ \\
C9 & $0.17350(19)$ & $0.4201(2)$ & $-0.14845(11)$ & $0.0462(5)$ \\
H9 & 0.1228 & 0.3690 & -0.1888 & $0.055^{*}$ \\
C12 & $-0.03366(17)$ & $0.2236(2)$ & $-0.08134(13)$ & $0.0463(4)$
\end{tabular}




$\begin{array}{lllll}\text { H12 } & -0.0337 & 0.2290 & -0.1386 & 0.056^{*} \\ \text { C2 } & 0.5060(2) & 0.7802(2) & 0.18111(14) & 0.0564(5) \\ \text { H2 } & 0.5441 & 0.8240 & 0.2291 & 0.068^{*} \\ \text { C8 } & 0.26887(19) & 0.5170(3) & -0.16981(11) & 0.0497(5) \\ \text { H8 } & 0.2832 & 0.5304 & -0.2253 & 0.060^{*} \\ \text { C15 } & -0.02974(17) & 0.2127(2) & 0.08669(13) & 0.0459(4) \\ \text { H15 } & -0.0286 & 0.2094 & 0.1441 & 0.055^{*} \\ \text { C6 } & 0.32011(15) & 0.56916(19) & -0.02768(10) & 0.0336(4) \\ \text { C11 } & 0.05559(15) & 0.3024(2) & -0.03164(11) & 0.0369(4) \\ \text { C1 } & 0.41594(19) & 0.6650(2) & 0.18517(12) & 0.0485(5) \\ \text { H1 } & 0.3938 & 0.6323 & 0.2370 & 0.058^{*} \\ \text { C14 } & -0.12109(18) & 0.1307(2) & 0.04107(15) & 0.0522(5) \\ \text { H14 } & -0.1802 & 0.0728 & 0.0673 & 0.063^{*} \\ \text { C13 } & -0.12326(18) & 0.1362(2) & -0.04368(15) & 0.0542(5) \\ \text { H13 } & -0.1841 & 0.0819 & -0.0756 & 0.065^{*} \\ \text { C3 } & 0.53854(19) & 0.8291(3) & 0.10520(14) & 0.0560(5) \\ \text { H3 } & 0.5994 & 0.9067 & 0.1008 & 0.067^{*} \\ \text { C7 } & 0.34327(18) & 0.5944(2) & -0.11007(11) & 0.0430(4) \\ \text { H7 } & 0.4068 & 0.6613 & -0.1244 & 0.052^{*}\end{array}$

Atomic displacement parameters $\left(\AA^{2}\right)$

\begin{tabular}{lllllll}
\hline & $U^{11}$ & $U^{22}$ & $U^{33}$ & $U^{12}$ & $U^{13}$ & $U^{23}$ \\
\hline Zn1 & $0.04271(14)$ & $0.03704(13)$ & $0.02811(12)$ & $0.00301(8)$ & $0.00414(9)$ & $0.00265(7)$ \\
C12 & $0.0482(3)$ & $0.0413(2)$ & $0.0446(3)$ & $0.00621(19)$ & $0.00184(19)$ & $0.00566(19)$ \\
C13 & $0.0765(4)$ & $0.0645(3)$ & $0.0410(3)$ & $0.0083(3)$ & $0.0181(2)$ & $-0.0013(2)$ \\
N1 & $0.0457(8)$ & $0.0353(8)$ & $0.0339(8)$ & $0.0016(6)$ & $0.0007(6)$ & $0.0008(6)$ \\
N2 & $0.0369(7)$ & $0.0307(7)$ & $0.0296(7)$ & $0.0045(6)$ & $0.0023(6)$ & $0.0007(5)$ \\
N3 & $0.0390(8)$ & $0.0358(8)$ & $0.0410(8)$ & $0.0042(6)$ & $0.0045(6)$ & $0.0028(6)$ \\
C4 & $0.0467(11)$ & $0.0434(10)$ & $0.0518(11)$ & $-0.0007(8)$ & $0.0073(9)$ & $0.0049(8)$ \\
C5 & $0.0361(9)$ & $0.0319(8)$ & $0.0381(9)$ & $0.0066(7)$ & $0.0039(7)$ & $0.0012(7)$ \\
C10 & $0.0390(9)$ & $0.0332(9)$ & $0.0330(9)$ & $0.0083(7)$ & $0.0005(7)$ & $-0.0021(7)$ \\
C9 & $0.0546(11)$ & $0.0507(11)$ & $0.0328(10)$ & $0.0051(9)$ & $-0.0013(8)$ & $-0.0075(8)$ \\
C12 & $0.0454(10)$ & $0.0379(10)$ & $0.0541(11)$ & $0.0058(8)$ & $-0.0071(8)$ & $-0.0063(8)$ \\
C2 & $0.0657(13)$ & $0.0434(11)$ & $0.0571(13)$ & $-0.0012(10)$ & $-0.0170(10)$ & $-0.0062(10)$ \\
C8 & $0.0623(12)$ & $0.0595(12)$ & $0.0284(9)$ & $0.0057(10)$ & $0.0120(8)$ & $0.0005(8)$ \\
C15 & $0.0426(10)$ & $0.0410(10)$ & $0.0549(11)$ & $0.0047(8)$ & $0.0101(8)$ & $0.0073(8)$ \\
C6 & $0.0359(9)$ & $0.0315(8)$ & $0.0335(9)$ & $0.0073(7)$ & $0.0045(7)$ & $0.0014(7)$ \\
C11 & $0.0370(9)$ & $0.0304(8)$ & $0.0429(10)$ & $0.0077(7)$ & $-0.0015(7)$ & $-0.0017(7)$ \\
C1 & $0.0646(12)$ & $0.0426(11)$ & $0.0370(10)$ & $0.0025(9)$ & $-0.0063(9)$ & $-0.0023(8)$ \\
C14 & $0.0384(10)$ & $0.0358(10)$ & $0.0831(16)$ & $0.0036(8)$ & $0.0102(10)$ & $0.0056(10)$ \\
C13 & $0.0402(10)$ & $0.0361(10)$ & $0.0844(16)$ & $0.0035(8)$ & $-0.0091(10)$ & $-0.0081(10)$ \\
C3 & $0.0493(11)$ & $0.0444(11)$ & $0.0731(15)$ & $-0.0086(9)$ & $-0.0050(10)$ & $-0.0010(10)$ \\
C7 & $0.0477(11)$ & $0.0461(11)$ & $0.0365(10)$ & $0.0045(8)$ & $0.0132(8)$ & $0.0037(8)$ \\
& & & & & &
\end{tabular}


Geometric parameters $\left(\AA,{ }^{\circ}\right)$

\begin{tabular}{|c|c|c|c|}
\hline $\mathrm{Zn} 1-\mathrm{N} 2$ & $2.1123(14)$ & C9-H9 & 0.9300 \\
\hline $\mathrm{Zn} 1-\mathrm{N} 3$ & $2.1893(16)$ & $\mathrm{C} 12-\mathrm{C} 11$ & $1.386(3)$ \\
\hline $\mathrm{Zn} 1-\mathrm{N} 1$ & $2.2160(17)$ & $\mathrm{C} 12-\mathrm{C} 13$ & $1.392(3)$ \\
\hline $\mathrm{Zn} 1-\mathrm{Cl} 3$ & $2.2684(8)$ & $\mathrm{C} 12-\mathrm{H} 12$ & 0.9300 \\
\hline $\mathrm{Zn} 1-\mathrm{Cl} 2$ & $2.2883(11)$ & $\mathrm{C} 2-\mathrm{C} 3$ & $1.365(3)$ \\
\hline $\mathrm{N} 1-\mathrm{C} 1$ & $1.336(2)$ & $\mathrm{C} 2-\mathrm{C} 1$ & $1.375(3)$ \\
\hline $\mathrm{N} 1-\mathrm{C} 5$ & $1.343(2)$ & $\mathrm{C} 2-\mathrm{H} 2$ & 0.9300 \\
\hline $\mathrm{N} 2-\mathrm{C} 6$ & $1.333(2)$ & $\mathrm{C} 8-\mathrm{C} 7$ & $1.379(3)$ \\
\hline $\mathrm{N} 2-\mathrm{C} 10$ & $1.341(2)$ & $\mathrm{C} 8-\mathrm{H} 8$ & 0.9300 \\
\hline $\mathrm{N} 3-\mathrm{C} 15$ & $1.335(2)$ & $\mathrm{C} 15-\mathrm{C} 14$ & $1.380(3)$ \\
\hline $\mathrm{N} 3-\mathrm{C} 11$ & $1.349(2)$ & C15-H15 & 0.9300 \\
\hline $\mathrm{C} 4-\mathrm{C} 5$ & $1.383(3)$ & $\mathrm{C} 6-\mathrm{C} 7$ & $1.393(2)$ \\
\hline $\mathrm{C} 4-\mathrm{C} 3$ & $1.384(3)$ & $\mathrm{C} 1-\mathrm{H} 1$ & 0.9300 \\
\hline $\mathrm{C} 4-\mathrm{H} 4$ & 0.9300 & $\mathrm{C} 14-\mathrm{C} 13$ & $1.374(3)$ \\
\hline $\mathrm{C} 5-\mathrm{C} 6$ & $1.487(2)$ & $\mathrm{C} 14-\mathrm{H} 14$ & 0.9300 \\
\hline $\mathrm{C} 10-\mathrm{C} 9$ & $1.392(3)$ & $\mathrm{C} 13-\mathrm{H} 13$ & 0.9300 \\
\hline $\mathrm{C} 10-\mathrm{C} 11$ & $1.487(2)$ & $\mathrm{C} 3-\mathrm{H} 3$ & 0.9300 \\
\hline $\mathrm{C} 9-\mathrm{C} 8$ & $1.378(3)$ & $\mathrm{C} 7-\mathrm{H} 7$ & 0.9300 \\
\hline $\mathrm{N} 2-\mathrm{Zn} 1-\mathrm{N} 3$ & $74.72(6)$ & $\mathrm{C} 11-\mathrm{C} 12-\mathrm{H} 12$ & 120.7 \\
\hline $\mathrm{N} 2-\mathrm{Zn} 1-\mathrm{N} 1$ & $74.08(5)$ & $\mathrm{C} 13-\mathrm{C} 12-\mathrm{H} 12$ & 120.7 \\
\hline $\mathrm{N} 3-\mathrm{Zn} 1-\mathrm{N} 1$ & $146.20(6)$ & $\mathrm{C} 3-\mathrm{C} 2-\mathrm{C} 1$ & $118.67(19)$ \\
\hline $\mathrm{N} 2-\mathrm{Zn} 1-\mathrm{Cl} 3$ & $143.70(4)$ & $\mathrm{C} 3-\mathrm{C} 2-\mathrm{H} 2$ & 120.7 \\
\hline $\mathrm{N} 3-\mathrm{Zn} 1-\mathrm{Cl} 3$ & $100.88(5)$ & $\mathrm{C} 1-\mathrm{C} 2-\mathrm{H} 2$ & 120.7 \\
\hline $\mathrm{N} 1-\mathrm{Zn} 1-\mathrm{Cl} 3$ & $96.58(5)$ & $\mathrm{C} 7-\mathrm{C} 8-\mathrm{C} 9$ & $120.90(17)$ \\
\hline $\mathrm{N} 2-\mathrm{Zn} 1-\mathrm{Cl} 2$ & $104.28(4)$ & $\mathrm{C} 7-\mathrm{C} 8-\mathrm{H} 8$ & 119.5 \\
\hline $\mathrm{N} 3-\mathrm{Zn} 1-\mathrm{Cl} 2$ & $97.98(5)$ & $\mathrm{C} 9-\mathrm{C} 8-\mathrm{H} 8$ & 119.5 \\
\hline $\mathrm{N} 1-\mathrm{Zn} 1-\mathrm{Cl} 2$ & $101.98(6)$ & $\mathrm{N} 3-\mathrm{C} 15-\mathrm{C} 14$ & $122.48(19)$ \\
\hline $\mathrm{C} 13-\mathrm{Zn} 1-\mathrm{Cl} 2$ & $111.99(2)$ & $\mathrm{N} 3-\mathrm{C} 15-\mathrm{H} 15$ & 118.8 \\
\hline $\mathrm{C} 1-\mathrm{N} 1-\mathrm{C} 5$ & $118.30(16)$ & $\mathrm{C} 14-\mathrm{C} 15-\mathrm{H} 15$ & 118.8 \\
\hline $\mathrm{C} 1-\mathrm{N} 1-\mathrm{Zn} 1$ & $126.03(13)$ & $\mathrm{N} 2-\mathrm{C} 6-\mathrm{C} 7$ & $121.20(16)$ \\
\hline $\mathrm{C} 5-\mathrm{N} 1-\mathrm{Zn} 1$ & $115.64(11)$ & $\mathrm{N} 2-\mathrm{C} 6-\mathrm{C} 5$ & $114.56(14)$ \\
\hline $\mathrm{C} 6-\mathrm{N} 2-\mathrm{C} 10$ & $121.12(14)$ & $\mathrm{C} 7-\mathrm{C} 6-\mathrm{C} 5$ & $124.22(16)$ \\
\hline $\mathrm{C} 6-\mathrm{N} 2-\mathrm{Zn} 1$ & $119.46(11)$ & $\mathrm{N} 3-\mathrm{C} 11-\mathrm{C} 12$ & $121.68(17)$ \\
\hline $\mathrm{C} 10-\mathrm{N} 2-\mathrm{Zn} 1$ & $118.66(11)$ & $\mathrm{N} 3-\mathrm{C} 11-\mathrm{C} 10$ & $115.04(15)$ \\
\hline $\mathrm{C} 15-\mathrm{N} 3-\mathrm{C} 11$ & $118.97(16)$ & $\mathrm{C} 12-\mathrm{C} 11-\mathrm{C} 10$ & $123.27(17)$ \\
\hline $\mathrm{C} 15-\mathrm{N} 3-\mathrm{Zn} 1$ & $125.12(13)$ & $\mathrm{N} 1-\mathrm{C} 1-\mathrm{C} 2$ & $123.08(19)$ \\
\hline $\mathrm{C} 11-\mathrm{N} 3-\mathrm{Zn} 1$ & $115.48(11)$ & $\mathrm{N} 1-\mathrm{C} 1-\mathrm{H} 1$ & 118.5 \\
\hline $\mathrm{C} 5-\mathrm{C} 4-\mathrm{C} 3$ & $119.01(18)$ & $\mathrm{C} 2-\mathrm{C} 1-\mathrm{H} 1$ & 118.5 \\
\hline $\mathrm{C} 5-\mathrm{C} 4-\mathrm{H} 4$ & 120.5 & $\mathrm{C} 13-\mathrm{C} 14-\mathrm{C} 15$ & $118.87(19)$ \\
\hline $\mathrm{C} 3-\mathrm{C} 4-\mathrm{H} 4$ & 120.5 & $\mathrm{C} 13-\mathrm{C} 14-\mathrm{H} 14$ & 120.6 \\
\hline $\mathrm{N} 1-\mathrm{C} 5-\mathrm{C} 4$ & $121.64(17)$ & $\mathrm{C} 15-\mathrm{C} 14-\mathrm{H} 14$ & 120.6 \\
\hline $\mathrm{N} 1-\mathrm{C} 5-\mathrm{C} 6$ & $114.90(15)$ & $\mathrm{C} 14-\mathrm{C} 13-\mathrm{C} 12$ & $119.42(19)$ \\
\hline $\mathrm{C} 4-\mathrm{C} 5-\mathrm{C} 6$ & $123.44(16)$ & $\mathrm{C} 14-\mathrm{C} 13-\mathrm{H} 13$ & 120.3 \\
\hline $\mathrm{N} 2-\mathrm{C} 10-\mathrm{C} 9$ & $120.56(17)$ & $\mathrm{C} 12-\mathrm{C} 13-\mathrm{H} 13$ & 120.3 \\
\hline $\mathrm{N} 2-\mathrm{C} 10-\mathrm{C} 11$ & $114.32(15)$ & $\mathrm{C} 2-\mathrm{C} 3-\mathrm{C} 4$ & $119.32(19)$ \\
\hline
\end{tabular}




$$
\begin{aligned}
& \text { C9-C10-C11 } \\
& \text { C8-C9-C10 } \\
& \text { C8-C9-H9 } \\
& \text { C10-C9-H9 } \\
& \text { C11-C12-C13 } \\
& \text { N2-Zn1-N1-C1 } \\
& \text { N3-Zn1-N1-C1 } \\
& \text { C13-Zn1-N1-C1 } \\
& \text { C12-Zn1-N1-C1 } \\
& \text { N2-Zn1-N1-C5 } \\
& \text { N3-Zn1-N1-C5 } \\
& \text { C13-Zn1-N1-C5 } \\
& \text { C12-Zn1-N1-C5 } \\
& \text { N3-Zn1-N2-C6 } \\
& \text { N1-Zn1-N2-C6 } \\
& \text { C13-Zn1-N2-C6 } \\
& \text { C12-Zn1-N2-C6 } \\
& \text { N3-Zn1-N2-C10 } \\
& \text { N1-Zn1-N2-C10 } \\
& \text { C13-Zn1-N2-C10 } \\
& \text { C12-Zn1-N2-C10 } \\
& \text { N2-Zn1-N3-C15 } \\
& \text { N1-Zn1-N3-C15 } \\
& \text { C13-Zn1-N3-C15 } \\
& \text { C12-Zn1-N3-C15 } \\
& \text { N2-Zn1-N3-C11 } \\
& \text { N1-Zn1-N3-C11 } \\
& \text { C13-Zn1-N3-C11 } \\
& \text { C12-Zn1-N3-C11 } \\
& \text { C1-N1-C5-C4 } \\
& \text { Zn1-N1-C5-C4 } \\
& \text { C1-N1-C5-C6 } \\
& \text { Zn1-N1-C5-C6 } \\
& \text { C3-C4-C5-N1 } \\
& \text { C3-C4-C5-C6 } \\
& \text { C6-N2-C10-C9 } \\
& \text { Zn1-N2-C10-C9 } \\
& \text { C6-N2-C10-C11 } \\
& \text { Zn1-N2-C10-C11 }
\end{aligned}
$$

$125.12(16)$

118.35 (18)

120.8

120.8

$118.6(2)$

$174.74(16)$

$151.48(14)$

30.57 (15)

$-83.62(15)$

$-7.44(11)$

$-30.69(17)$

$-151.60(11)$

$94.20(11)$

$177.72(13)$

$10.88(11)$

$90.10(13)$

$-87.75(12)$

$-12.17(11)$

$-179.01(13)$

$-99.79(13)$

$82.36(12)$

$-177.15(15)$

$-153.97(13)$

$-34.18(14)$

80.15 (14)

$10.46(11)$

33.64 (17)

$153.42(11)$

$-92.24(11)$

$0.1(2)$

$-177.91(13)$

$-178.31(15)$

$3.69(18)$

$0.3(3)$

$178.53(17)$

$1.4(2)$

$-168.50(13)$

$-178.11(14)$

11.94 (18)
$\mathrm{C} 2-\mathrm{C} 3-\mathrm{H} 3$

$\mathrm{C} 4-\mathrm{C} 3-\mathrm{H} 3$

$\mathrm{C} 8-\mathrm{C} 7-\mathrm{C} 6$

$\mathrm{C} 8-\mathrm{C} 7-\mathrm{H} 7$

$\mathrm{C} 6-\mathrm{C} 7-\mathrm{H} 7$

$\mathrm{N} 2-\mathrm{C} 10-\mathrm{C} 9-\mathrm{C} 8$

$\mathrm{C} 11-\mathrm{C} 10-\mathrm{C} 9-\mathrm{C} 8$

$\mathrm{C} 10-\mathrm{C} 9-\mathrm{C} 8-\mathrm{C} 7$

$\mathrm{C} 11-\mathrm{N} 3-\mathrm{C} 15-\mathrm{C} 14$

$\mathrm{Zn} 1-\mathrm{N} 3-\mathrm{C} 15-\mathrm{C} 14$

$\mathrm{C} 10-\mathrm{N} 2-\mathrm{C} 6-\mathrm{C} 7$

$\mathrm{Zn} 1-\mathrm{N} 2-\mathrm{C} 6-\mathrm{C} 7$

$\mathrm{C} 10-\mathrm{N} 2-\mathrm{C} 6-\mathrm{C} 5$

$\mathrm{Zn} 1-\mathrm{N} 2-\mathrm{C} 6-\mathrm{C} 5$

$\mathrm{N} 1-\mathrm{C} 5-\mathrm{C} 6-\mathrm{N} 2$

$\mathrm{C} 4-\mathrm{C} 5-\mathrm{C} 6-\mathrm{N} 2$

N1-C5-C6-C7

$\mathrm{C} 4-\mathrm{C} 5-\mathrm{C} 6-\mathrm{C} 7$

$\mathrm{C} 15-\mathrm{N} 3-\mathrm{C} 11-\mathrm{C} 12$

$\mathrm{Zn} 1-\mathrm{N} 3-\mathrm{C} 11-\mathrm{C} 12$

$\mathrm{C} 15-\mathrm{N} 3-\mathrm{C} 11-\mathrm{C} 10$

$\mathrm{Zn} 1-\mathrm{N} 3-\mathrm{C} 11-\mathrm{C} 10$

$\mathrm{C} 13-\mathrm{C} 12-\mathrm{C} 11-\mathrm{N} 3$

$\mathrm{C} 13-\mathrm{C} 12-\mathrm{C} 11-\mathrm{C} 10$

$\mathrm{N} 2-\mathrm{C} 10-\mathrm{C} 11-\mathrm{N} 3$

C9- $10-\mathrm{C} 11-\mathrm{N} 3$

$\mathrm{N} 2-\mathrm{C} 10-\mathrm{C} 11-\mathrm{C} 12$

C9- $\mathrm{C} 10-\mathrm{C} 11-\mathrm{C} 12$

$\mathrm{C} 5-\mathrm{N} 1-\mathrm{C} 1-\mathrm{C} 2$

$\mathrm{Zn} 1-\mathrm{N} 1-\mathrm{C} 1-\mathrm{C} 2$

$\mathrm{C} 3-\mathrm{C} 2-\mathrm{C} 1-\mathrm{N} 1$

N3-C15-C14-C13

$\mathrm{C} 15-\mathrm{C} 14-\mathrm{C} 13-\mathrm{C} 12$

$\mathrm{C} 11-\mathrm{C} 12-\mathrm{C} 13-\mathrm{C} 14$

$\mathrm{C} 1-\mathrm{C} 2-\mathrm{C} 3-\mathrm{C} 4$

$\mathrm{C} 5-\mathrm{C} 4-\mathrm{C} 3-\mathrm{C} 2$

$\mathrm{C} 9-\mathrm{C} 8-\mathrm{C} 7-\mathrm{C} 6$

N2-C6-C7-C8

$\mathrm{C} 5-\mathrm{C} 6-\mathrm{C} 7-\mathrm{C} 8$
120.3

120.3

117.84 (17)

121.1

121.1

$-0.5(3)$

179.05 (16)

$-0.8(3)$

$0.2(3)$

$-171.91(14)$

$-1.2(2)$

$168.70(12)$

$177.68(14)$

$-12.46(18)$

$5.3(2)$

$-173.10(16)$

$-175.93(16)$

5.7 (3)

$0.0(2)$

172.94 (13)

$179.28(14)$

$-7.83(18)$

-0.2 (3)

$-179.42(16)$

$-2.3(2)$

$178.21(16)$

$176.96(15)$

$-2.6(3)$

$-0.4(3)$

$177.41(15)$

$0.2(3)$

$-0.3(3)$

$0.1(3)$

$0.2(3)$

$0.1(3)$

-0.4 (3)

1.1 (3)

-0.1 (3)

-178.83 (16)

Hydrogen-bond geometry $\left(A,{ }^{\circ}\right)$

\begin{tabular}{lllll}
\hline$D-\mathrm{H} \cdots A$ & $D-\mathrm{H}$ & $\mathrm{H} \cdots A$ & $D \cdots A$ & $D-\mathrm{H} \cdots A$ \\
\hline $\mathrm{C} 4-\mathrm{H} 4 \cdots \mathrm{Cl} 2^{\mathrm{i}}$ & 0.93 & 2.68 & $3.518(2)$ & 151 \\
$\mathrm{C} 13-\mathrm{H} 13 \cdots \mathrm{Cl} 2^{\mathrm{ii}}$ & 0.93 & 2.81 & $3.686(2)$ & 158 \\
\hline
\end{tabular}

Symmetry codes: (i) $-x+1,-y+1,-z$; (ii) $-x,-y,-z$. 